\title{
Uterine Dehiscence
}

National Cancer Institute

\section{Source}

National Cancer Institute. Uterine Dehiscence. NCI Thesaurus. Code C114282.

Asymptomatic separation of the uterine wall, usually at the site of a prior uterine scar, that does not include the overlying serosa. 